\title{
Acolhimento com classificação de risco para a Estratégia Saúde da Família : a prática em uma unidade docente-assistencial
}

\author{
Embracement with risk classification for the Family Health Strategy: the practice in a teaching health care centre \\ Acogimiento con calificación de riesgo para la Estrategia de Salud Familiar: la práctica en un servicio docente-asistencial
}

Alessandro da Silva Scholze. Universidade do Vale do Itajaí (UNIVALI). Itajaí, SC, Brasil. alescholze@gmail.com (Autor correspondente)

\section{Resumo}

0 acolhimento é visto, concomitantemente, como tecnologia do encontro, criando redes de conversações para resgatar um enfoque centrado no usuário, e como ação técnico-assistencial para reorganizar os processos de trabalho. Neste artigo, apresenta-se uma reflexão teórica sobre as possibilidades de empregar formas de Acolhimento com Classificação de Risco (ACR) na Estratégia Saúde da Família (ESF), a partir da descrição de sua implantação no processo de trabalho de três equipes da ESF que atuam em uma mesma Unidade de Saúde (US) em Itajaí-SC. A construção coletiva de novos fluxos de atenção e instrumentos auxiliares para a classificação de risco pode ser empregada para qualificar o serviço segundo os princípios da equidade e integralidade. As limitações do processo devem ser identificadas e discutidas como parte de constante reavaliação e reajuste no processo de trabalho. 0 ACR mostrou-se um desafio e uma possibilidade de avanço no sentido da humanização pela horizontalização das relações e busca de corresponsabilização tanto no interior das ESF como nos contatos destas com os usuários.

\section{Abstract}

User Embracement is conceived as a technology to establish network relations and rescue a user oriented approach on health services, at the same time that it develops actions towards the reorganization of the working processes of health professionals. This article presents a theoretical reflection regarding the possibility of applying an Embracement Health Risk Classification Tool (EHRC-tool) for the Family Health Strategy (FHS), by describing its implementation in the working process of three family health teams working at the same health centre in Itajai, Santa Catarina state. The collective construction of new pathways of attention and support tools for risk classification can be applied to qualify the service according to the principles of equity and comprehensiveness. The limitations of the process should be identified and discussed as part of constant reassessment and adjustment in the work process. The implementation of the EHRC-Tool presents many challenges and possibilities for improving a humanistic approach by levelling the relationship and seeking co-responsibility both within the FHS and in the daily contact with users.

\section{Resumen}

El acogimiento se ve como una tecnología del encuentro, creando redes de conversaciones para rescatar un enfoque centrado en el usuario, y como una actividad técnico-asistencial para reorganizar los procesos de trabajo en salud. En este artículo se presenta una reflexión teórica sobre las posibilidades de utilizar el Acogimiento con Calificación de Riesgo (ACR) en la Estrategia de Salud Familiar (ESF), a partir de la descripción de su aplicación en el proceso de trabajo de tres equipos de la ESF que actúan en una misma Unidad de Salud (US) en Itajaí, Santa Catarina. La construcción colectiva de nuevos flujos de atención y de instrumentos auxiliares para la calificación de riesgo puede ser empleada para calificar el servicio según los principios de equidad e integralidad. Las limitaciones del proceso deben ser identificadas y discutidas como parte de reevaluación constante y reajuste en el proceso de trabajo. El ACR se mostró como un desafío y una oportunidad para avanzar hacia la humanización de las relaciones, buscando la corresponsabilidad tanto en los equipos como en sus contactos con los usuarios.
Palavras-chave: Acolhimento Estratégia Saúde da Família Acesso aos Serviços de Saúde Serviços de Integração Docente-Assistencial

\section{Keywords:}

User Embracement Family Health Strategy Health Services Accessibility Teaching Care Integration Services

\section{Palabras clave:} Acogimiento Estrategia de Salud Familiar Accesibilidad a los Servicios de Salud Servicios de Integración Docente Asistencial 


\section{Introdução}

A implantação do Sistema Único de Saúde (SUS) foi defendida por Sérgio Arouca como um processo civilizatório contemporâneo da sociedade brasileira, em constante mudança pelos embates entre sua vocação para interferir amplamente nas condiçóes de vida da população e as resistências do trabalho em saúde fragmentado. A Política Nacional de Humanização (PNH) conjugou esforços para superar essas resistências, propondo mudar a cultura dos envolvidos no sistema, aumentando o grau de corresponsabilização de usuários e profissionais, tanto na atenção à saúde como na gestão dos processos de trabalho. ${ }^{1}$

$\mathrm{Na}$ Atenção Primária à Saúde (APS), os parâmetros para acompanhamento da implantação da PNH incluem "[...] formas de acolhimento e inclusão do usuário que promovam a otimizaçáo dos serviços, o fim das filas, a hierarquizaçáo de riscos e o acesso aos demais níveis do sistema." ${ }^{2} \mathrm{O}$ acolhimento é visto, concomitantemente, como uma tecnologia do encontro ao buscar a inclusão do usuário e do profissional em uma rede de conversaçôes capaz de resgatar o enfoque do trabalho em saúde centrado no usuário; e como ação técnico-assistencial, proposta para reorganizar os processos de trabalho, ofertando uma atenção integral e ampliando a capacidade da equipe multiprofissional para identificar e resolver problemas de saúde. ${ }^{3-5}$

A atenção à demanda espontânea é um dos desafios das equipes da Estratégia Saúde da Família (ESF), pois as expectativas na sua implantação voltam-se, em geral, para atividades de promoção e proteção da saúde, idealmente com grupos homogêneos como gestantes ou hipertensos. O cotidiano das equipes é, entretanto, marcado pela busca de soluçóes para problemas interpretados como urgências/emergências pelos usuários e suas famílias, gerando discordâncias e conflitos entre estes e as equipes ou mesmo entre os diferentes profissionais. ${ }^{6} \mathrm{O}$ dilema entáo posto é como organizar o processo de trabalho da ESF de modo a lidar adequadamente com as situaçóes emergentes da demanda espontânea sem que esta absorva recursos e tempo necessários para a construção de um espaço dedicado à demanda programada no processo de trabalho dos profissionais da APS, pois a capacidade de gerenciar retornos permite utilizar a demora permitida de modo eficiente, contribuindo para estabelecer vínculos a partir da longitudinalidade das açóes assistenciais.

$\mathrm{Na}$ prática corrente, as respostas à demanda espontânea são pontuais, voltadas para o que se notabilizou como "apagar incêndios", à custa do desgaste de todos os envolvidos e, mesmo, da eficácia dos esforços empreendidos. Assim, cada evento não agendado exige a criação de um fluxo ad hoc para acessar os recursos humanos e/ou materiais necessários, nem sempre efetivo. Em outros casos, tal rede já está informalmente sedimentada, conforme as disposiçóes e humores de cada profissional, com o consequente risco para as relaçóes entre os membros da equipe e a possível sobrecarga daqueles que se mostram mais acessíveis ou resolutivos.

Uma alternativa possível é a construção coletiva de um processo de trabalho em que as funçóes de cada membro de equipe estejam claramente definidas, tanto nas situaçóes de demanda espontânea como programada, de modo a valorizar as competências clínicas de todos. Neste processo, as redes de conversaçôes devem ser de conhecimento comum, fluindo a um só tempo de forma flexível para permitir uma aproximação com situações inesperadas, e de modo recorrente, para evitar o esforço repetitivo de gerar canais de comunicação a cada demanda que chega à Unidade de Saúde (US).

Conduziu-se aqui uma reflexão sobre as possibilidades de empregar formas de Acolhimento com Classificação de Risco (ACR) na ESF, a partir da descrição de sua implantação no processo de trabalho de três equipes da ESF que atuam na mesma US em Itajaí, Santa Catarina.

\section{Descrição da experiência}

O processo de trabalho na US na qual o autor do presente artigo atua como médico em uma equipe da ESF, dava-se com foco na demanda programada, a partir das agendas dos médicos e enfermeiras. Os profissionais da ESF atendiam usuários sem agendamento prévio quando a demanda espontânea era considerada prioritária para que fosse negociado um atendimento médico imediato. Esse acesso fora da agenda era fortuito, proveniente da avaliação na recepçáa pelas auxiliares administrativas, auxiliares/técnicas de enfermagem, enfermeiras ou mesmo dos Agentes Comunitários de Saúde (ACS), conforme o usuário morasse ou não na área adscrita e de acordo com a existência de vínculo com os profissionais com que entrasse em contato na chegada ao serviço.

As três equipes constituíram-se como parte de um Distrito Docente-Assistencial (DDA) em 2005, mediante convênio entre Secretaria Municipal de Saúde e a Universidade do Vale do Itajaí-SC, responsáveis pela cobertura da APS nas áreas adjacentes ao campus de Itajaí onde estavam sediadas. Cada equipe era responsável por populaçóes adscritas com cerca de 4000 pessoas, excedendo o ideal previsto para a ESF, sendo que as microáreas mais próximas correspondiam às de maior risco social, com casas construídas em terrenos invadidos no morro, ao redor do Presídio Regional. 
$\mathrm{Na}$ mesma unidade funcionavam ambulatórios de nível secundário, mantidos pelo curso de medicina, além de estágios de fonoaudiologia, psicologia, enfermagem, nutrição, odontologia e fisioterapia. Apesar das possibilidades de interdisciplinaridade e maior resolutividade na integração com esse meio acadêmico, as demandas trazidas por esses estágios eram também fonte de sobrecarga para os profissionais das equipes, além de consumir maior tempo nas ações em saúde envolvendo discentes na unidade ou no território.

Entre as propostas do DDA, estavam o desenvolvimento e a divulgação de tecnologias em saúde, realizavam-se reuniōes semanais, das quais participavam os médicos e enfermeiras das equipes, também docentes dos cursos de medicina e enfermagem da Universidade.

Nesses encontros, os profissionais identificaram a necessidade de qualificação do acesso ao serviço. Este apresentava limitaçôes em diferentes aspectos: geográficos, sócio-organizacionais e da disponibilidade de serviços, não estando formalizado nenhum fluxo de atendimento a situações de urgência/emergência. ${ }^{5}$ Mesmo excluindo estas situaçóes, demandas por consultas no mesmo dia constituem um desafio para a APS em diferentes países. Apesar da possibilidade de reduzir retornos agendados, um médico responsável pelo atendimento a mais de 1000 pessoas terá dificuldades para prover cuidados adequados, calculando-se que, para atender 2000 pessoas, ele terá de trabalhar 17,4 horas por dia. Essa relação poderia ser melhorada com a delegação de tarefas a outros profissionais, bem como por formas de acesso além da consulta, como telefone ou e-mail. ${ }^{7}$

No DDA, diante da necessidade de discussão caso a caso com o médico, nada garantia que o usuário tivesse suas condiçóes de saúde adequadamente avaliadas por um profissional de saúde, nem que estas determinariam de maneira equânime a mobilização dos recursos necessários. Tais situaçôes geram respostas negativas, agendamentos de longo prazo capazes de comprometer a resolutividade do serviço e o estabelecimento de vínculos, além da possibilidade de transferência das demandas para outras unidades, incluindo serviços de pronto-atendimento, mesmo sem necessidade do ponto de vista técnico. ${ }^{8,9}$

Inicialmente, médicos e enfermeiras buscaram um referencial relativo ao acolhimento como forma de superar limitaçóes no acesso. A partir das discussões teóricas e da socialização de diferentes protocolos e experiências na implantação do acolhimento, o grupo verificou que seria necessária alguma forma de classificação de risco para um acolhimento resolutivo, permitindo hierarquizar as demandas. ${ }^{10}$

Além da busca por maior satisfação e segurança para responder às demandas dos usuários, a opção pelo ACR surgiu também do reconhecimento de nossas limitações: não haver sempre médicos na US e horários de funcionamento em dois turnos, da manhã e à tarde, diferente de um serviço de pronto-atendimento. Mesmo em sistemas consolidados com APS forte, como no Reino Unido, identificam-se as limitaçóes para acesso aos serviços somente em horário comercial, bem como a possibilidade de maior procura por pronto-atendimento quando não se consegue agendamento nas unidades de APS. ${ }^{11}$

Isso conduziu à necessidade de se estabelecer uma linguagem comum aos profissionais envolvidos, de modo que a avaliaçáo inicial das condiçôes de saúde não ficasse centrada no médico, mas que os critérios para defini-las fossem previamente conhecidos por todos. Para tanto, foram identificados protocolos já estabelecidos em outros serviços, os quais foram discutidos quanto à sua aplicabilidade para a realidade local. ${ }^{12-14}$

O ACR foi sistematizado como uma ferramenta para serviços de pronto-atendimento, mas mostrava-se uma opção interessante, uma vez que o instrumento buscado para orientar as condutas iniciais no acolhimento deveria reunir algumas qualidades: ser de fácil visualização; permitir a abordagem das situações mais comuns no serviço; incluir critérios objetivos verificáveis tanto pelos profissionais médicos como pelos de enfermagem; definir critérios suficientemente sensíveis para não exceder à demora permitida em casos potencialmente graves e também suficientemente específicos para não comprometer o atendimento à demanda programada. ${ }^{2}$

A seguir, o grupo incluiu as auxiliares/técnicas de enfermagem nas reunióes, identificando-as como os membros da equipe já naturalmente envolvidos no primeiro contato com os usuários na unidade, conduzindo as pré-consultas e realizando procedimentos diversos. Eram, assim, informantes-chave para a identificação das situaçôes mais comuns, a fim de convencionar quais parâmetros seriam utilizados no ACR. Estes foram selecionados a partir dos protocolos já discutidos, ${ }^{12-14}$ gerando os critérios para categorização dos riscos que guiariam as condutas das auxiliares/técnicas de enfermagem, como responsáveis primárias pelo acolhimento aos usuários. Resumidamente, os encaminhamentos das demandas espontâneas seguiriam o fluxo apresentado na Figura 1. 


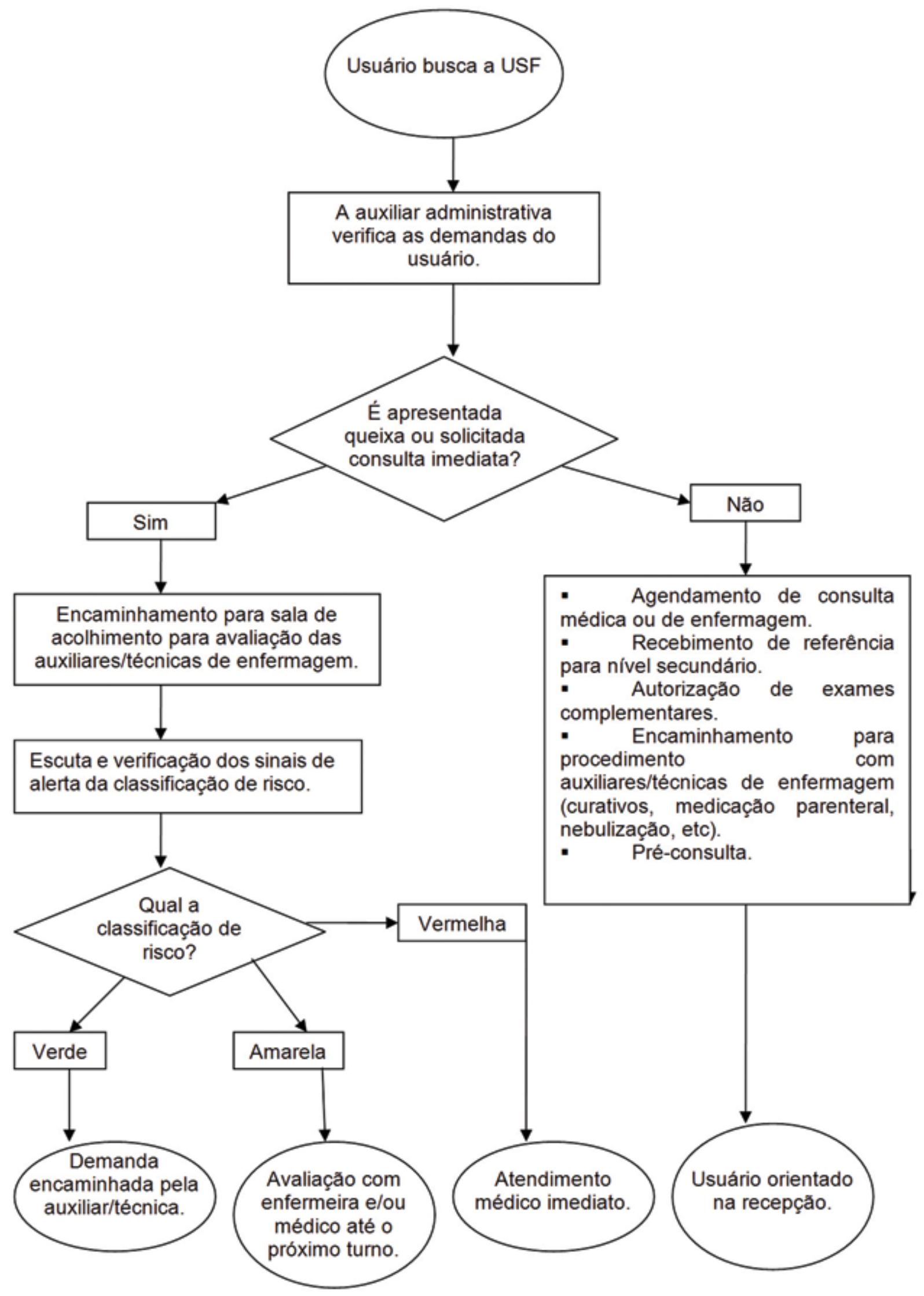

Figura 1. Fluxo do Acolhimento com Classificação de Risco na ESF. 
Os ACS participaram da implantaçáo do acolhimento a partir das reuniōes semanais de suas respectivas equipes e as informaçóes trazidas por eles foram utilizadas para validar as demandas identificadas pelos demais profissionais como potencialmente comuns nas situaçôes de acolhimento. Ainda, os ACS foram encarregados de divulgar entre os usuários a nova forma de acesso ao serviço.

Os critérios da classificação de risco foram selecionados a partir dos protocolos consultados, ${ }^{12-14}$ considerando-se as especificidades da demanda local discutidas ao longo do processo de implantação do acolhimento. Foram utilizadas até o momento três versóes desse instrumento, sendo que a última é apresentada no Quadro 1, no formato disponível para consulta pelas auxiliares/técnicas de enfermagem.

Quadro 1. Critérios para Acolhimento com Classificação de Risco na ESF.

\begin{tabular}{|c|c|c|}
\hline Verde & Amarelo & Vermelho (Sinais Vitais) \\
\hline Renovação de receitas & Trauma/Violência & Idade: até 28 dias \\
\hline Atestados/declarações & Amenorreia: avaliação da enfermagem (Beta - HCG) & $\begin{array}{l}\text { Frequência respiratória: } \\
\text { adulto> } 24 \\
\text { criança> } 40\end{array}$ \\
\hline Tosse (Produtiva $>3$ semanas: ex. escarro) & Risco para DST/HIV: avaliação da enfermagem & $\begin{array}{l}\text { Frequência cardíaca: } \\
>100 \text { ou <45bpm }\end{array}$ \\
\hline \multirow{4}{*}{ Lesões de pele $>48 \mathrm{~h}$} & Intensidade da dor $<7$ & Intensidade da dor $\geq 7$ \\
\hline & Vômitos/Diarreia > $48 \mathrm{~h}$ & Vômitos/Diarreia $<48 \mathrm{~h}$ \\
\hline & Sangramento único/cessado & Sangramento recorrente/em curso \\
\hline & & Temperatura axilar $\geq 37,8^{\circ} \mathrm{C}$ ou $<35,5^{\circ} \mathrm{C}$ \\
\hline
\end{tabular}

As situaçôes que determinassem necessidade de atendimento médico foram diferenciadas conforme a demora permitida, estabelecida a partir dos critérios de ACR, da seguinte forma:

- Verde - sem critérios de risco: orientação pela auxiliar/técnica de enfermagem e, quando necessário, o usuário terá atendimento eletivo com médico ou enfermeira conforme agenda.

- Amarelo - critérios de risco presentes sem risco imediato: o usuário tem avaliação com a enfermeira no mesmo turno e/ou avaliação médica com demora permitida até o próximo turno.

- Vermelho - critérios de risco imediato: o usuário tem avaliação médica imediata, sendo conduzido à sala de medicação.

O sinal vital "dor" era verificado com o uso de uma escala analógica que estabelecia ausência de dor como zero e a pior dor já sentida como 10, permitindo o registro de níveis intermediários entre estes. ${ }^{15} \mathrm{Na}$ presença de um dos indicadores de risco vermelho, o profissional que estivesse conduzindo o acolhimento deveria levar a pessoa até a sala de medicação e informar um dos médicos disponíveis para atendê-la assim que possível nesse local, de modo a náo retardar as condutas necessárias e também evitar situaçóes de tensão na sala de espera diante do "passar na frente" dos demais usuários.

As situações não previstas no ACR ou que gerassem dúvidas para as auxiliares/técnicas de enfermagem deveriam ser levadas à atenção de uma das enfermeiras, preferencialmente aquela responsável pela área do usuário atendido. Na ausência destas, o caso deveria ser discutido com um dos médicos, também preferencialmente membro da ESF responsável pela área do usuário atendido. Se nenhum destes estivesse disponível, o caso seria levado aos membros de uma das outras ESF, de forma que o atendimento por médico ou enfermeira de uma ESF diferente daquela à qual o usuário era adscrito não fosse um obstáculo para o acesso. Esse apoio entre equipes da mesma US favorece estabelecer vínculo com o usuário, que tem acesso garantido ao serviço, sem prejuízo de atividades na comunidade ou reuniōes das equipes. ${ }^{6,10} \mathrm{Na}$ experiência da US do Saco Grande, em Florianópolis, foi criada a figura das "equipes irmãs", que dão cobertura recíproca aos usuários da outra área, na ausência dos profissionais da área de adscrição. Essa informação foi obtida em comunicação feita pessoalmente com a coordenadora da UBS Saco Grande, enfermeira Marly Wuerges Aquino, durante visita técnica realizada pelo autor em 2012. 
Essa é uma forma de lidar com os momentos de ausência de profissionais de uma determinada equipe, sem assumir a opção de escalar uma "equipe de acolhimento" entre várias na mesma US, que seria responsável por toda demanda não agendada em um determinado período de tempo, independente da adscriçáo dos usuários. ${ }^{16} \mathrm{O}$ processo de trabalho no acolhimento deve manter como orientação o direcionamento das pessoas para suas equipes de adscrição, estimulando o vínculo e longitudinalidade, ainda que sempre surjam situações nas quais os profissionais deverão acolher pessoas "fora de área", sem que esse seja um motivo para bloquear o acesso, mas sim um momento para uma referência adequada. ${ }^{10}$

\section{Discussão}

Durante todos os momentos de discussão no grupo, buscou-se a clareza conceitual quanto ao acolhimento, como atitude, por ser um elemento transversal e indispensável ao longo dos contatos do usuário com os profissionais e que suas facetas técnico-assistenciais deveriam ser empregadas na perspectiva de acolhimento mútuo entre os profissionais, atuando com "portas abertas" entre si, permitindo a discussão de dúvidas que inevitavelmente surgiriam na sua implantação. Tais dúvidas foram consideradas como inerentes ao $\mathrm{ACR}$, que não foi pensado para ser exaustivo na inclusão dos possíveis problemas de saúde trazidos pelos usuários, facilitando sua aplicação no cotidiano dos profissionais responsáveis pelo acolhimento. ${ }^{2,4}$

Depois do início das atividades de acolhimento na unidade, as ESF mantiveram um espaço de reavaliação permanente, em reuniôes mensais com médicos, enfermeiras, auxiliares/técnicas de enfermagem e auxiliar administrativo, sendo as informaçóes repassadas para os ACS nas reunióes de cada equipe e, quando considerado necessário pelo grupo, os ACS também participariam desses momentos de discussão do acolhimento.

Nas discussões mensais, as dificuldades vivenciadas no desenvolvimento das atividades de acolhimento partiram, na maioria das vezes, das auxiliares/técnicas de enfermagem. Tais dificuldades podem ser entendidas no contexto do processo de trabalho, pois estas profissionais assumiram o papel central como responsáveis pelo acolhimento, necessitando resgatar competências clínicas pouco exigidas no processo de trabalho mais centrado no médico até então. Situações similares foram verificadas em diferentes contextos de implantação do acolhimento, mesmo que tais competências estejam de acordo com a lei do exercício profissional da enfermagem. . $^{3,17,18}$

As dificuldades muitas vezes diziam respeito a inseguranças no encaminhamento das demandas dos pacientes com classificação de risco verde ou amarela, sem avaliação imediata dos médicos. Isso foi abordado no grupo reafirmando a sensibilidade dos sinais de risco empregados, o que foi validado empiricamente pelas equipes, na medida em que verificavam que os usuários tinham suas demandas adequadamente atendidas segundo o conceito de demora permitida, conferindo progressivamente maior confiança nesse processo de trabalho.

Ainda assim, os esforços das equipes para manter uma rede de conversaçôes comum entre os profissionais tiveram de ser renovados constantemente. Isso se deveu a várias substituiçóes entre aqueles profissionais, gerando novas "capacitaçóes em serviço" para cada profissional ingressante, especialmente no caso daqueles sem formação direcionada para participar da avaliação e tomada de decisões no cuidado aos usuários. ${ }^{10}$ Nesses momentos, a existência de um protocolo consolidado entre os membros mais antigos foi visto como um facilitador para a inclusão dos novos profissionais no processo de trabalho já estabelecido.

Outro desafio predominante na vivência das auxiliares/técnicas de enfermagem na implantação do ACR envolveu a percepção inicial da falta de reconhecimento de seu trabalho pelas pessoas atendidas. Estas profissionais relatavam situaçóes de usuários descontentes ao não serem vistos imediatamente pelo médico, considerando o ACR apenas uma barreira a mais no acesso, em uma perspectiva de pronto-socorro, o que pode ser observado em serviços que buscam oferecer outras opçóes além da consulta médica. ${ }^{19,20}$ Por outro lado, ao longo do tempo, o reconhecimento positivo dos usuários em relação ao atendimento realizado pelas auxiliares/técnicas e a disponibilidade dos demais profissionais para dar suporte às suas ações foram essenciais para lidar com isso.

Mesmo superando a distribuição de fichas na porta, o acolhimento na ESF pode manter uma lógica médico-centrada, resultando na falta de acesso ao atendimento clínico e cardápios limitados em possibilidades de referência, sendo que o simples aumento do número de equipes não garante ampliação de acesso caso os profissionais mantenham a visão da ESF como dirigida aos excluídos, sem necessidade de maiores investimentos. As dificuldades na implantação por vezes limitam o ACR a uma "triagem humanizada", quando não há inserção de toda a equipe no processo ou mantêm-se excedentes populacionais por equipe. ${ }^{20-22}$

As dificuldades podem ser enfrentadas pela valorização das competências clínicas de todos os membros da equipe, provendo o devido suporte e orientação, com protocolos previamente definidos que garantam maior autonomia e capacidade de intervenção. Esse processo é favorecido quando há uma implantação participativa, envolvendo colegiados locais e 
representantes do nível central, além da própria ESF, garantindo aos profissionais uma maior qualificaçáo no seu trabalho e na produção do cuidado por meio da educação permanente e da manutenção de proporçôes adequadas de população adscrita por equipe. ${ }^{3,4,23}$

Por outro lado, todos os profissionais reconheceram a necessidade de se conduzir os acolhimentos com maior agilidade, sem comprometer a escuta qualificada, mas evitando longas esperas para os usuários, tanto nas demandas espontâneas como programadas. Verificavam-se diferentes graus de detalhamento das queixas e dos critérios de risco nos registros feitos pelas auxiliares/técnicas de enfermagem, mesmo pela inexistência do hábito destas registrarem seus atendimentos nos prontuários. ${ }^{16}$ Criou-se, entáo, um Boletim de Acolhimento, a ser preenchido e anexado ao prontuário, também permitindo o registro quando a pessoa atendida náo possuía prontuário na unidade. Este boletim detalhava os itens mínimos a serem verificados no acolhimento, padronizando a coleta dos dados necessários para o encaminhamento do usuário a outros profissionais, sem exigir que a avaliação inicial fosse completamente repetida, além de proporcionar uma fonte de dados para posterior avaliação do processo.

A presente reflexão sobre o ACR na ESF partiu de um contexto, infelizmente, comum à APS no Brasil, com populações adscritas que excedem o ideal por equipe e sofrem de carências de natureza intersetorial, mas encontram na ampliação do acesso às US talvez seu único contato direto com os serviços públicos. Tal situação gera demandas sempre crescentes para os recursos humanos e materiais limitados da ESF. Qualquer processo de acolhimento conduz a um julgamento de valor que pode ser entendido como classificaçáo de risco, explícita ou não, pois os encaminhamentos da escuta qualificada (caso exista) geram hierarquização das demandas e conduzirão o usuário a diferentes itinerários, de resolutividades variáveis, conforme as necessidades percebidas pelo profissional e as possibilidades oferecidas pelo sistema local.

Quando o profissional em questão não é o médico, mas um enfermeiro sem experiência na assistência da APS ou técnico de enfermagem, como na prática em questão, o ACR pode ser visto como um recurso didático para os profissionais, ao assumirem um papel mais ativo no acolhimento que descentra o trabalho do médico, e tranquilizador para o profissional e usuários, uma vez que permite identificar os quadros de maior risco biomédico, sem prejuízo de uma avaliação das vulnerabilidades no contexto da pessoa. Uma vez garantidos, naquele momento, o acesso longitudinal e a escuta qualificada, criam-se condiçôes para ampliar o foco sobre a pessoa, respondendo às suas demandas que surjam como queixas somáticas e, concomitantemente, trabalhando os aspectos de disponibilidade ou carência de recursos que compóem as vulnerabilidades do usuário, superando o caráter individual de risco, que é o objeto bastante delimitado do instrumento de classificação utilizado. ${ }^{24}$

\section{Considerações finais}

Conduzir o ACR de forma explícita torna mais transparentes para profissionais e usuários os processos de tomada de decisão empregados, ao se definir como serão utilizados os recursos limitados para prover cuidados em saúde. Além do reconhecimento do profissional para com o sofrimento do usuário, possibilita-se assim que as pessoas atendidas construam seus vínculos com o serviço a partir de respostas positivas a suas demandas. Abre-se ainda uma oportunidade de ampliar a visão do usuário para as outras pessoas que buscam o serviço concomitantemente com seus próprios problemas, permitindo que estes também se reconheçam mutuamente, fomentando a corresponsabilização.

As diversas experiências com acolhimento na ESF identificadas na literatura podem ser vistas como oportunidades de avançar no sentido de ampliar as ofertas de cuidados das US. ${ }^{25}$ Neste estudo, restringiu-se o acolhimento como um primeiro momento do contato do usuário com o serviço, sem discutir as conhecidas limitaçóes do sistema relativas à integraçáo e comunicação entre diferentes níveis e setores. Entretanto, se reconhecem as potencialidades do acolhimento para a criação de espaços coletivos e desmedicalizantes, para profissionais e usuários, a serem descritos e pesquisados, bem como uma nova fonte a ser integrada nas açóes de prevenção e vigilância da ESF, desde que conte com registros consistentes que ampliem a perspectiva das equipes a partir da demanda espontânea que chega à US até os determinantes de saúde encontrados no seu entorno.

A proposta de ACR aqui discutida alinha-se aos esforços da $\mathrm{PNH}$, identificando-se com as peculiaridades do próprio SUS. Ela foi conduzida com vistas a ser permanentemente reavaliada e remodelada conforme as respostas dos usuários e profissionais às açôes implementadas, sem considerá-las definitivas apenas por terem sido postas em prática. Tal perspectiva de reflexão permanente está intrinsecamente ligada à integração do acolhimento no processo de trabalho em saúde, visto que a humanização dos serviços de saúde passa pela horizontalização das relaçôes e pela busca de corresponsabilização tanto no interior das equipes de saúde como nos contatos destas com os usuários. 


\section{Referências}

1. Pasche DF, Passos E, Hennington EA. Cinco anos da política nacional de humanização: trajetória de uma política pública. Ciênc Saúde Coletiva [Internet]. 2011 [acesso em 2013 Sep 10];16(11):4541-48. Disponível em: http://www.scielosp.org/pdf/csc/v16n11/a27v16n11.pdf

2. Ministério da Saúde (BR), Secretaria-Executiva, Núcleo Técnico da Política Nacional de Humanização. HumanizaSUS: acolhimento com avaliação e classificação de risco: um paradigma ético estético no fazer em saúde [Internet]. Brasília: Ministério da Saúde; 2004 [acesso em 2008 Aug 15]. Disponível em: http://bvsms.saude.gov.br/bvs/publicacoes/acolhimento.pdf. 48p. (Textos Básicos de Saúde; Série B).

3. Oliveira LML, Tunini ASM, Silva FC. Acolhimento: concepções, implicações no processo de trabalho e na atenção em saúde. Revista APS [Internet]. 2008 [acesso em 2013 Sep 10];11(4):362-73. Disponível em: http://www.aps.uff..br/index.php/aps/article/viewArticle/394

4. Santos IMV, Santos AM. Acolhimento no Programa Saúde da Família: revisão das abordagens em periódicos brasileiros. Rev Salud Pública. 2011;13(4):703-16. http://dx.doi.org/10.1590/S0124-00642011000400015

5. Pereira RPA. O acolhimento e a Estratégia Saúde da Família. Grupo de estudos em Saúde da Família [Internet]. Belo Horizonte: AMMFC; 2006 [acesso em 2009 Apr 14]. Disponível em: http://www.smmfc.org.br/gesf/RPAP_acolhimento_esf.htm

6. Santos TVC, Penna CMM. Daily demands in primary care: the view of health professionals and users. Texto Contexto Enferm. 2013;22(1):149-56. http://dx.doi.org/10.1590/S0104-07072013000100018

7. Bodenheimer T, Pham HH. Primary Care: current problems and proposed solutions. Health Affairs [Internet]. 2010 [acesso em 2013 Aug 27];29(5):799-805. Disponível em: http://dx.doi.org/10.1377/hlthaff.2010.0026

8. Nery AA, Carvalho CGR, Santos FPA, Nascimento MS, Rodrigues VP. Saúde da Família: visão dos usuários. Rev enferm UERJ. 2011;19(3):397-402

9. Urbano GB. Integralidade na prática assistencial da equipe de enfermagem: a relação da escuta na interação usuário e profissional no município de Paranavaí - PR [dissertação]. Rio de Janeiro: Universidade do Estado do Rio de Janeiro; 2009. Português.

10. Tesser CD, Poli Neto P, Campos GWS. Acolhimento e (des)medicalização social: um desafio para as equipes de saúde da família. Ciênc Saúde Colet. 2010;15(Suppl 3):3615-24. http://dx.doi.org/10.1590/S1413-81232010000900036

11. 11. Kontopantelis E, Roland M, Reeves D. Patient experience of access to primary care: identification of predictors in a national patient survey. BMC Family Practice [Internet]. 2010 [acesso em 2013 May 10];11:61. Disponível em: http://dx.doi.org/10.1186/1471-2296-11-61

12. 12. Campos GWS, Carpintero MC, Bueno JMS. Protocolo de Acolhimento da Secretaria Municipal de Saúde de Campinas. Campinas; 2001. Português.

13. Secretaria Municipal da Saúde de São Paulo. $2^{\circ}$ Caderno de apoio ao acolhimento: orientações, rotinas e fluxos sob a ótica do risco/ vulnerabilidade [Internet]. São Paulo: Prefeitura do Município de São Paulo; 2004 [acesso em 2006 Aug 23]. Disponível em: http://www6. prefeitura.sp.gov.br/secretarias/saude/publicacoes/0054

14. Mayema MA, editor. A Construção do Acolhimento: a proposta de mudança do processo de trabalho em saúde do município de Bombinhas. Bombinhas: Secretaria Municipal de Saúde de Bombinhas; 2007.

15. Schoeller MT. Guia prático para o tratamento da dor oncológica. $2^{a}$ ed. Florianópolis: Sociedade Brasileira de Oncologia Clínica; 2002.

16. Barreto Junior OS, Santos EF. Acolhimento na Estratégia Saúde da Família. In: Sociedade Brasileira de Medicina de Família e Comunidade (SBMFC), editor. Porto Alegre: Artmed Panamericana; 2006. p. 43-64. (Programa de Atualização em Medicina de Família e Comunidade, Ciclo 3 , Módulo 3).

17. Pinafo E, Lima JVC, Baduy RS. Acolhimento: concepção dos auxiliares de enfermagem e percepção de usuários em uma unidade de saúde da família. Espaç saúde [Internet]. 2008 Jun [acesso em 2008 Aug 15];9(2):17-25. Disponível em: http://www.ccs.uel.br/espacoparasaude/v9n2/Artigo\%2053-2007\%20_Editado_.pdf

18. Brasil. Decreto n. 94.406 de 8 de junho de 1987. Dispõe sobre a regulamentação do exercício da Enfermagem e dá outras providências. Diário Oficial da União. 1987; Seção 1: 8853-8855. Português.

19. Souza ECF, Vilar RLA, Rocha NSPD, Uchoa AC, Rocha PM. Acesso e acolhimento na atenção básica: uma análise da percepção dos usuários e profissionais de saúde. Cad Saúde Pública 2008;24(Suppl 1):S100-10. http://dx.doi.org/10.1590/S0102-311X2008001300015

20. Baraldi DC, Souto BGA. A demanda do Acolhimento em uma Unidade de Saúde da Família em São Carlos, São Paulo. Arq Bras Ciências Saúde. $2011 ; 36(1): 10-7$

21. Hennington EA. Acolhimento como prática interdisciplinar num programa de extensão universitária. Cad Saúde Pública 2005;21(1):256-65. http://dx.doi.org/10.1590/S0102-311X2008001300015

22. Coelho MO, Jorge MSB, Araújo ME. O acesso por meio do acolhimento na atenção básica à saúde. Rev Baiana Saúde Pública. 2009 Jul-Set;33(3):440-52.

23. Vieira-da-Silva LM, Esperidião MA, Viana SV, Alves VS, Lemos DVS, Caputo MC et al. Avaliação da implantação de programa voltado para melhoria da acessibilidade e humanização do acolhimento aos usuários na rede básica. Salvador, 2005-2008. Rev Bras Saúde Matern Infant. 2010 Nov;10(Suppl 1):S131-43. http://dx.doi.org/10.1590/S1519-38292010000500012

24. Sánchez AIM, Bertolozzi MR. Pode o conceito de vulnerabilidade apoiar a construção do conhecimento em Saúde Coletiva? Ciênc Saúde Colet. 2007;12(2):319-24. http://dx.doi.org/10.1590/S1413-81232007000200007

25. Mitre SM, Andrade EIG, Cotta RMM. Avanços e desafios do acolhimento na operacionalização e qualificação do Sistema Único de Saúde na Atenção Primária: um resgate da produção bibliográfica do Brasil. Ciênc Saúde Colet. 2012;17(8):2071-85.

http://dx.doi.org/10.1590/S1413-81232012000800018 